045

\section{Depots of solubilised elastin promote the formation of blood vessels and elastic fibres in rat}

W.F. Daamen ${ }^{1}$, S.T.M. Nillesen ${ }^{1}$, R. Wismans ${ }^{1}$, D. Reinhardt ${ }^{2}$, T. Hafmans ${ }^{1}$, J.H. Veerkamp ${ }^{1}$, T.H. van Kuppevelt ${ }^{1}$

${ }^{1}$ Dept. of Biochemistry 280, NCMLS, Radboud University Nijmegen Medical Centre, Nijmegen, The Netherlands ${ }^{2}$ Dept. of Anatomy and Cell Biology, Faculty of Medicine and Faculty of Dentistry, McGill University, Montreal, Canada

\section{Summary}

Porous biomaterials composed of highly purified collagen and solubilised elastin were prepared by freezing and lyophilisation and studied by subcutaneous implantation in young Sprague Dawley rats. The scaffolds, containing depots of soluble elastin, showed no calcification in this sensitive calcification model, in contrast to scaffolds containing insoluble elastin fibres. Scaffolds induced the formation of blood vessels and promoted elastic fibre synthesis as was shown with specific antibodies.

\section{Introduction}

Elastin is the prime protein in tissues that display elasticity of e.g. lung, aorta, and skin. Upon injury, elastic fibres are not readily replaced which causes a delay in tissue regeneration. Incorporation of solubilised elastin (obtained from insoluble elastin fibres by hydrolysis) into biomaterials may improve tissue regeneration, since solubilised elastin is able to promote fibroblast proliferation as well as elastin synthesis [1-3].

\section{Experimental methods}

Elastin fibres were hydrolysed by means of oxalic acid [4]. Three different scaffolds were prepared composed of: collagen $(\mathrm{COL})[5,6]$, collagen + elastin fibres $\left(\mathrm{COL}-\mathrm{EL}_{\text {fibre }}\right)[7,8]$, and collagen + solubilised elastin $\left(\mathrm{COL}-\mathrm{EL}_{\mathrm{sol}}\right)$. All scaffolds were chemically crosslinked and morphologically and biochemically characterised. Scaffolds were subcutaneously implanted in 3 weeks old Sprague Dawley rats, and retrieved after 3, 7, and 21 days. Paraffin sections of explants were stained with haematoxylin-eosin; elastin and collagen were stained according to Elastin Van Gieson; calcium deposits were visualized by Von Kossa staining. Cryo-sections were stained for type IV collagen, rat elastin and the elastin fibre-associated molecule fibrillin using specific antibodies (immunohistochemistry).

\section{Results and discussion}

Porous biomaterials composed of collagen and solubilised elastin could be prepared by freezing and lyophilising. Solubilised elastin was associated with the collagen fibrils of the scaffolds. Over time, solubilised elastin was released from the $\mathrm{COL}-\mathrm{EL}_{\mathrm{sol}}$ scaffold as indicated by reduced Elastin-Van
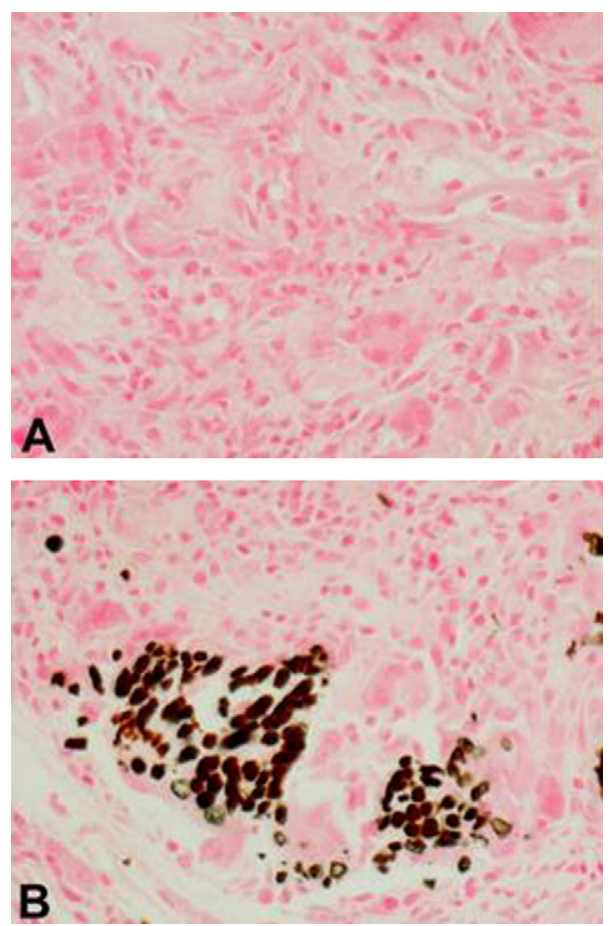

Fig. 1. Light microscopical evaluation of the calcification of crosslinked scaffolds, 21 days after implantation in young Sprague Dawley rats for (A) COL-ELsol and (B) COL-ELfibre scaffolds. Calcium deposits have an intense black colour due to Von Kossa staining. In COL-ELfibre, deposits were only associated with elastin fibres.

Gieson staining. However, at day 21 after implantation, substantial staining was still present in the scaffolds, indicating a slow release mechanism.

Blood vessels were most abundant in COL- $\mathrm{EL}_{\text {sol }}$ scaffolds, both at the periphery and within the scaffold as analysed by type IV collagen staining. These scaffolds also contained more large blood vessels than $\mathrm{COL}-\mathrm{EL}_{\text {fibre }}$ and COL. In COL-EL fibre, vascularisation was considerably less. In COL, blood vessels were found only at the periphery of the scaffolds.

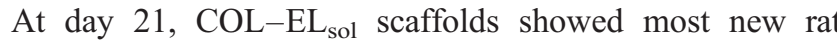
elastin, not only in blood vessels, but also as thin elastic fibres within the scaffold. In COL-EL fibre scaffolds, new elastin was found mainly in blood vessels present in the capsule around the implant and small amounts of thin elastic fibres were sporadically found at the periphery of the scaffolds.

In COL scaffolds, rat elastin was present only in blood vessels in the capsule. In blood vessels, rat elastin co-localised with fibrillin-1 and fibrillin-2 staining, but only fibrillin-1 staining co-localised with the thin elastic fibres.

COL-EL $\mathrm{El}_{\mathrm{sol}}$ scaffolds did not calcify during the time course of the experiment in contrast to $\mathrm{COL}-\mathrm{EL}_{\text {fibre }}$ scaffolds (Fig. 1). In $\mathrm{COL}-\mathrm{EL}_{\text {fibre }}$ scaffolds, elastin fibres calcified from day 7 onwards, whereas the collagen fibrils did not. Scaffolds composed of only collagen did not calcify.

\section{Conclusion}

COL-EL $\mathrm{E}_{\text {sol }}$ scaffolds provide a non-calcifying release vehicle for solubilised elastin that stimulate angiogenesis and 
elastic fibre formation and have great potential for soft tissue engineering.

\section{References}

[1] Kamoun, A., Landeau, J.M., Godeau, G., Wallach, J., Duchesnay, A., Pellat, B., Hornebeck, W. Growth stimulation of human skin fibroblasts by elastin-derived peptides, Cell Adhes. Commun. 3 (4) (1995) 273-281.

[2] Fülöp Jr. T., Jacob, M.P., Khalil, J., Wallach, J., Robert, L. Biological effects of elastin peptides, Pathol Biol (Paris) 46 (7) (1998) 497-506.

[3] Hinek, A., Wang, Y., Liu, K., Mitts, T.F., Jimenezet, F. Proteolytic digest derived from bovine Ligamentum Nuchae stimulates deposition of new elastin-enriched matrix in cultures and transplants of human dermal fibroblasts, J. Dermatol. Sci. (2005)

[4] Partridge, S.M., Davis, H.F., Adairet, G.S. The chemistry of connective tissues. 2-Soluble proteins derived from partial hydrolysis of elastin, Biochem. J. 61 (1) (1955) 11-21.

[5]. Pieper, J.S., Oosterhof, A., Dijkstra, P.J., Veerkamp, J. H., Kuppevelt, van T.H. Preparation and characterization of porous crosslinked collagenous matrices containing bioavailable chondroitin sulphate, Biomaterials 20 (1999) 847-858.

[6] Pieper, J.S., Hafmans, T., Veerkamp, J.H., Kuppevelt, van T.H. Development of tailor-made collagen-glycosaminoglycan matrices: EDC/NHS crosslinking, and ultrastructural aspects, Biomaterials 21 (6) (2000) 581-593.

[7] Daamen, W.F., Hafmans, T., Veerkamp, J.H., Kuppevelt, van T.H. Isolation of intact elastin fibers devoid of microfibrils, Tissue Eng 11 (7-8) (2005) 1168-1176.

[8] Daamen, W.F., Moerkerk, van H.Th.B., Hafmans, T., Buttafoco, L., Poot, A.A., Veerkamp, J.H., Kuppevelt, van T.H. Preparation and evaluation of molecularly-defined collagenelastin-glycosaminoglycan scaffolds for tissue engineering, Biomaterials 24 (22) (2003) 4001-4009.

doi:10.1016/j.jconrel.2006.09.062

046

Rheological and mechanical properties of Pluronic-alginate gels for drug-eluting stent coating

G. Grassi ${ }^{1}$, E. Noro ${ }^{2}$, R. Farra ${ }^{1}$, G. Guarnieri ${ }^{1}$, R. Lapasin $^{2}$, M. Grassi $^{2}$, P. Matricardi ${ }^{3}$, T. Coviello ${ }^{3}$, A. DalCortivo ${ }^{3}$, F. Alhaique $^{3}$

${ }^{1}$ Department of Internal Medicine, University Hospital of Trieste, Cattinara I-34149, Trieste, Italy

${ }^{2}$ Department of Chemical Engineering DICAMP, University of Trieste, Piazzale Europa 1, I-34127, Trieste, Italy

${ }^{3}$ Faculty of Pharmacy, University of Rome "La Sapienza", Piazzale A. Moro 5, I-00185, Rome, Italy

\section{Summary}

In this study, the preliminary results on the mechanical and rheological properties of a new delivery system based on Pluronic and alginate are reported. The system described is specifically designed to carry into the blood vessel a new antiproliferative molecule representing an original approach to in-stent restenosis therapeutic treatment.

\section{Introduction}

Percutaneous transluminal angioplasty (PTA) has been used in the past to treat artery occlusion (stenosis) due to atherosclerotic plaques, mechanically removing the endovascular plaque. Because of the frequent re-occlusion (restenosis) of the treated vessel [1], bare-metal stents nowadays are usually placed in the artery lumen at the site of stenosis, significantly improving the clinical outcome of treated patients.

Indeed, the frequent induction of the exuberant proliferation of coronary smooth muscle cells (CSMCs), determines the reocclusion of the treated vessel (in-stent restenosis) [2,3,4] (Fig. 1). This problem has been partially overcome by the use of drugeluting stents (DES) which are able to deliver in situ antiproliferative drugs. Recently we focused our attention on a novel kind of antiproliferative molecules termed small interfering RNAs (siRNAs) [5]. These molecules need to be complexed with liposomes to cross cellular membranes efficiently in order to exert their therapeutic effect. To carry and deliver such liposomes locally, the gelling matrix made up by Pluronic F127 (PF127) and alginate has been proposed as a suitable endo-arterial delivery system. In fact, PF127 and alginate, separately, are able to form reverse thermoresponsive hydrogels and temperature insensitive ionotropic gels respectively. The innovative therapeutic procedure

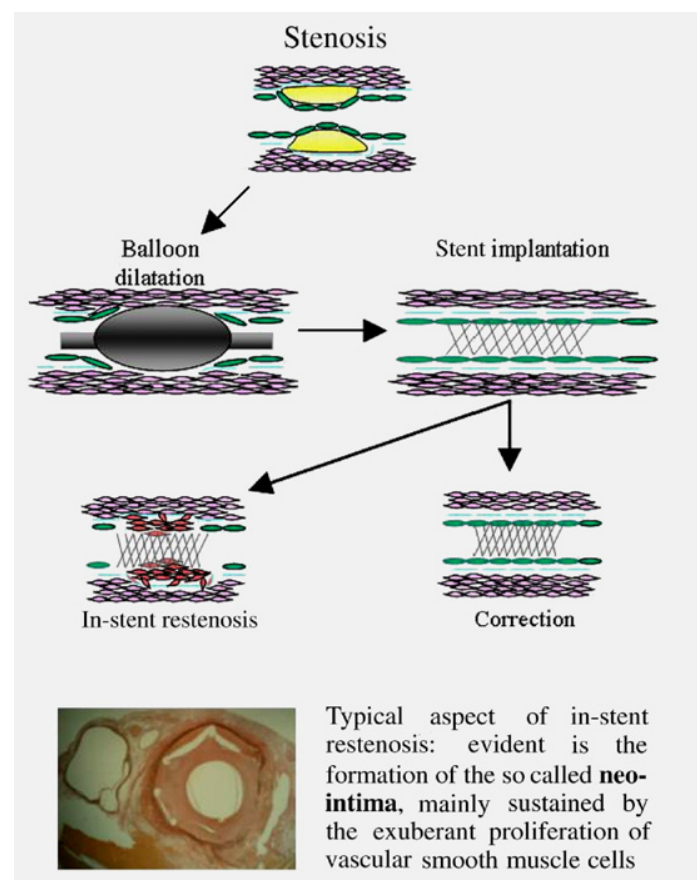

Fig. 1. Schematic representation of in-stent restenosis. 